\title{
Estado Atual de Conhecimento de Dinâmica de Populações
}

\author{
Current State of Population Dynamics Knowledge
}

\author{
Anna Clara Balbina Silva ; Afonso Pelli*a \\ ${ }^{a}$ Universidade Federal do Triângulo Mineiro. MG, Brasil. \\ *E-mail: apelli@terra.com.br
}

\begin{abstract}
Resumo
Compreender os mecanismos que regulam a dinâmica das populações espacialmente estruturadas é um desafio crítico para os ecólogos e gestores de conservação. A dinâmica de populações é um ramo da ecologia que estuda as populações como sistema em atividades, relacionando as influências ambientais com a distribuição e abundância dos indivíduos e suas interações com o ambiente. O presente artigo é uma revisão bibliográfica, com o objetivo de identificar produções científicas relevantes sobre dinâmica populacional. Para isso, foram utilizados periódicos revisados por pares, na base de Periódicos Capes. A pesquisa foi realizada em junho de 2019, utilizando-se as palavras-chave para título contendo: "population dynamics" e no assunto "ecology", a partir de 2014, quando o texto completo estava disponível. Foram considerados como critérios de exclusão os artigos publicados antes de 2014. Após a leitura dos títulos dos artigos, foram selecionados 34 artigos que foram lidos na íntegra. Em livros disponíveis no acervo da biblioteca da Universidade Federal do Triângulo Mineiro, foram selecionados quatro livros no tema dinâmica populacional. O referencial teórico aborda os aspectos da dinâmica de populações, tabela de vida, formas de crescimento e interações populacionais. Ressalta-se a necessidade de novos estudos que ainda possuem lacunas, que venha complementar e contribuir para o conhecimento de organismos que faltam ou ainda não possuem registros de estudos.
\end{abstract}

Palavras-chave: Taxas de Natalidade e Mortalidade. Atributos Populacionais. Dispersão.

\begin{abstract}
Understanding the mechanisms that regulate the dynamics of spatially structured populations is a critical challenge for ecologists and conservation managers. Population dynamics is a branch of ecology that studies populations as a system in activities, relating environmental influences to the individuals' distribution and abundance and their interactions with the environment. This article is a bibliographic review, aiming to identify relevant scientific productions about population dynamics. Thus. peer-reviewed journals were used in the Capes Periodicals base, the research was conducted in June 2019, using the keywords for title containing "population dynamics" and in the subject "ecology", from 2014, when the full text was available. Exclusion criteria were: articles published before 2014, after reading the article titles, 34 articles were selected that met the initially proposed criteria and were read in full. In books available in the library collection of the Federal University of Triangulo Mineiro, with a search for the dynamic population theme, 4 books were used. The theoretical framework addresses the aspects of population dynamics, life table, forms of growth and population interactions. It is emphasized the need for further studies that still have gaps, which will complement and contribute to the knowledge of organisms that are missing or do not have study records.
\end{abstract}

Keywords: Birth and Mortality Rates. Population Attributes. Dispersion.

\section{Introdução}

O termo dinâmica de população foi utilizado pela primeira vez em 1925, por Lotka e Volterra. O modelo Presa-Predador que descreve as interações entre duas espécies, em que uma dessas (presa) dispõe de alimentos em abundância e a outra espécie (predador) tem como suprimento alimentar a população de presas (GAVINA et al., 2018). Mais tarde, Nicholson e Bailey na década de 1930 descreveram a dinâmica populacional de um sistema parasitoide/hospedeiro (HASSAN et al., 2018).

Outros autores como Silva e Oliveira (2018) sugerem que a dinâmica de população foi usada antes, em 1798, por Thomas Malthus, que publicou em seu artigo "An essay on the principle of population as it affects the future improvement of society", que discutia sobre o crescimento populacional e a produção de alimentos.
Vale ressaltar que na natureza se observam vários níveis de organização: organismos, indivíduos, populações, comunidades, ecossistemas e biosfera. Cada um desses níveis de organização possuiu atributos próprios. Resumidamente, pode-se citar que os organismos possuem um tegumento ou membrana que separa o meio interno do externo, mantendo as condições internas, mais ou menos constantes. O indivíduo seria a unidade de dispersão (DALL et al., 2012; SUTER II et al., 2005). Em insetos sociais, por exemplo, uma operária não se reproduz, dessa forma, apesar de ser um organismo, não é um indivíduo. O indivíduo é a unidade de dispersão, ou o formigueiro (PRADEU, 2016).

A população é o conjunto de indivíduos em determinada área, ao mesmo tempo. Uma das características dessa unidade de organização é que herdam genes de gene pool comum e, assim, os descendentes apresentam continuidade espacial e 
temporal (SOBY, 2017).

A dinâmica de populações é o estudo das populações como sistema em atividade e trata das influências ambientais sobre a população, como, por exemplo, os efeitos da temperatura e umidade (RICKFLES, 2010). Compreender os mecanismos que regulam a dinâmica de populações, com propriedades peculiares, é um dos objetivos para ecólogos que trabalham nesse nível de organização dos sistemas naturais (FERREIRA et al., 2019).

Processos populacionais internos, como nascimentos e mortes, ocorrem em nível local, enquanto processos externos, como a dispersão, ocorrem em nível interpopulacional (RICKFLES, 2010). Sendo assim, a dinâmica de população quantifica o quanto essa cresce e decresce no tempo, indica as etapas no ciclo de vida dos indivíduos, quanto à sobrevivência ou natalidade, que são os períodos críticos para esse aumento ou declínio do número de indivíduos, bem como alterações na estrutura das populações (CAYUELA et al., 2018).

Com base nessas considerações, o objetivo desta pesquisa foi identificar os estudos que abordam dinâmica populacional. A importância reside em fornecer um cenário sobre o que é pesquisado em dinâmica populacional, evidenciando o estado atual do conhecimento na área.

\section{Desenvolvimento}

\subsection{Metodologia}

Foi realizada uma pesquisa bibliográfica, com o objetivo de identificar produções científicas sobre dinâmica populacional. Para isso, foram utilizados periódicos revisados por pares, na base de Periódicos Capes. A pesquisa foi realizada em junho de 2019, sendo utilizadas as palavras-chave para título contendo: "population dynamics" e no assunto "ecology", a partir de 2014, quando o texto completo estava disponível. Os critérios de exclusão foram os artigos publicados antes de 2014. Foram encontrados 236 artigos, após a leitura dos títulos dos artigos se notou que alguns desses não preenchiam os critérios deste estudo, pois abordavam outras áreas do conhecimento, e não ecologia; como Ciências Sociais, Geográficas e Humanas. Foram selecionados 50 artigos para a leitura do resumo e foram excluídos os que não diziam respeito ao propósito deste estudo. Após a leitura dos resumos, foram selecionados 34 artigos que preenchiam os critérios inicialmente propostos (ecologia e dinâmica de populações) e que foram lidos na íntegra. Também foi realizada uma busca em livros disponíveis no acervo da biblioteca da Universidade Federal do Triângulo Mineiro sobre o tema: dinâmica populacional. Foram encontrados seis livros e, após a leitura, foram notadas que algumas informações se repetiam, dessa forma, se fez a seleção da edição mais recente, resultando em quatro livros.

\subsection{Aspectos da dinâmica de populações}

Uma população é definida como um agrupamento de indivíduos que pertencem a mesma espécie e que vivem em uma mesma área, ao mesmo tempo, e se relacionam com fatores ambientais que moldam as variações da população no tempo e no espaço (RICKFLES, 2010). Do mesmo modo que os indivíduos apresentam seus atributos, a população também apresenta alguns atributos específicos, como, por exemplo, a densidade, a taxa de natalidade, de mortalidade, a estrutura etária, entre outros (COSTA et al., 2016) Um aspecto importante da população é que os indivíduos que a compõe compartilham uma história adaptativa comum ao ambiente, assim a tendência geral é que o parentesco (e herança de alelos) entre indivíduos da mesma população é maior que de populações distintas (LEITÃO et al., 2018; RODRIGUESSOARES et al., 2018).

A metapopulação pode ser definida como uma rede formada por populações espacialmente estruturadas em agrupamentos, no qual os indivíduos se reproduzem localmente e, no qual a migração entre as populações influencia a dinâmica local (LANDA, 2008). Existem alguns modelos de metapopulação como o modelo clássico de Levins (1969), seguido pelo modelo de metapopulação espacialmente implícito de Roughgarden (1997) e pelo modelo de metapopulação espacialmente estruturado de Ovaskainen e Hanski (2001).

A densidade é a medida do número de indivíduos em relação a alguma unidade de espaço ou volume (DIAS, 2018). Pode ser absoluta, como o número de indivíduos por metro quadrado; ou relativa, que é a proporção de uma categoria taxonômica em relação à comunidade, por exemplo (LANDA, 2008).

A densidade sofre flutuações com nascimentos e mortes que ocorrem dentro da população, ou entrada e saída de indivíduos na população (WALTER, 2018). A taxa de crescimento de uma população depende de sua estrutura etária, assim, a taxa de natalidade e mortalidade são atributos que correspondem aos números de nascimentos e mortes em um determinado período (MEDEIROS et al., 2017). Em um estudo que contempla plantas jovens se percebeu que apesar da importante influência dos indivíduos jovens na dinâmica populacional das espécies arbóreas de florestas tropicais, as plantas, em fases iniciais de desenvolvimento, apresentam a taxa de mortalidade mais elevada que as plantas em fases maduras ou de maior tamanho (SOUZA et al.,2018).

Outro atributo é a dispersão, que pode influenciar a aptidão, a dinâmica populacional, o fluxo gênico, a deriva genética e a estrutura genética de populações (GOMES, 2018). Mesmo pequenas diferenças na dispersão podem alterar as interações ecológicas e desencadear uma cascata evolutiva (SOLOMON, 1976).

A saída dos indivíduos se denomina de emigração e afeta a população da mesma forma que a mortalidade, no caso da entrada de indivíduos se denomina de imigração e afeta a população da mesma forma que a natalidade (SANTOS et al., 2017). A dispersão também permite o resgate demográfico, quando populações deprimidas ou extirpadas 
são recolonizadas por imigrantes do resto da metapopulação (SILVA, 2007). Por outro lado, altas taxas de dispersão podem sincronizar a dinâmica das populações e aumentar o risco de extinção de toda a metapopulação (DIAS, 2018).

A dispersão entre manchas efêmeras e espacialmente isoladas pode ser arriscada e difícil (GOMES, 2018). Entre os riscos se incluem não encontrar habitat adequado ou não encontrar parceiros adequados e esses riscos são exacerbados entre indivíduos pequenos e relativamente imóveis, como insetos, ácaros e pequenos animais terrestres (SANTOS et al., 2107). Alguns animais resolveram esse problema de dispersão com o comportamento chamado de forésia, na qual um indivíduo utiliza de um outro para o transporte sem se prejudicar. A forésia também pode ser definida como um caso específico do comensalismo (MAIA et al., 2018).

\subsection{Tabelas de vida}

A relação entre os indivíduos de uma população e o meio ambiente pode ser expressa através das tabelas de vida. As tabelas de vida são uma ferramenta básica para descrever e avaliar a dinâmica populacional (MEDEIROS et al., 2017). Como é difícil certificar paternidade em muitas espécies, as tabelas de vida são normalmente baseadas nas fêmeas (COSTA et al., 2016). Os dados para confecção da tabela de vida são coletados acompanhando um grupo de indivíduos desde o momento em que nascer até a morte do último indivíduo (tabela de vida dinâmica) ou estimando a idade dos indivíduos através de uma característica particular, como anéis de crescimento, de chifres, de desgaste dos dentes, escamas, ou outro índice confiável (tabela de vida estática) (GIRÃO FILHO et al., 2018).

Em seus estudos, Marques et al. (2015) concluíram, através das informações da tabela de vida, que uma determinada espécie de predador, Euseius concordis, apresentou um crescimento populacional satisfatório quando trocada sua dieta e um ciclo de desenvolvimento curto, além da sua taxa intrínseca de crescimento ( $\mathrm{rm}$ ) ser superior ao da presa. Indicando, dessa forma, que ele pode ter alto potencial de controle de Tetranychus bastosi, uma praga do pinhão-manso.

A tabela de vida dinâmica acompanha um grupo de indivíduos nascidos, no mesmo período, desde o nascimento até a morte do último indivíduo. Já a tabela de vida estática considera a sobrevivência dos indivíduos, com a idade previamente conhecida (AKCA et al., 2015).

A tabela de vida dinâmica também possibilita estimar a taxa intrínseca de crescimento de uma população ou taxa exponencial de crescimento (r), assumida por uma população, com uma distribuição etária estável (CRUZ et al., 2016). As condições do ambiente influenciam as taxas intrínsecas de crescimento e, o desempenho dos indivíduos reflete na tabela de vida como a probabilidade de sobrevivência e a taxa de fecundidade, específicas por classe etária (MEDEIROS et al., 2017).

Portanto, a tabela de vida e a taxa intrínseca de crescimento respondem as variações nas condições do ambiente (SILVA et al., 2018). Dessa forma, populações distintas da mesma espécie, em condições ambientais distintas, irão apresentar atributos também distintos (FERREIRA et al., 2019).

\subsection{Formas de Crescimento Populacional}

O crescimento populacional é decorrente de dois fenômenos opostos, a natalidade e a mortalidade, aos quais é possível acrescentar a emigração e imigração. A variação no número de indivíduos em uma população pode ser positiva ou negativa (FONTANA et al., 2015). Se nada houvesse para frear o crescimento das populações, esse ocorreria em progressão exponencial ou geométrica. $\mathrm{O}$ número de indivíduos dependeria apenas do potencial biótico da população, ou seja, de sua capacidade reprodutiva. Porém, isso não ocorre na realidade, pois a partir de um determinado momento, o ambiente começa a opor resistência a esse crescimento e essa resistência aumenta à medida que o número de indivíduos também aumenta (PELLI et al. 2007).

As populações apresentam padrões característicos de aumento que são denominados formas de crescimento, representados por modelos matemáticos e por suas respectivas representações gráficos ou curvas de crescimento (BARROS et al., 2018). As curvas de crescimento são representações de variação do tamanho de uma população no tempo. Para fins de comparação, Thomas Malthus que discutia sobre o crescimento populacional, delineou as curvas de crescimento em modelo exponencial e o modelo logístico foi concebido por Verhulst (SILVA et al., 2018).

A população que tem crescimento exponencial aumenta em proporção ao seu próprio tamanho, portanto, ganha indivíduos cada vez mais rápido à medida que a população aumenta (BARROS et al., 2018).

Quando ocorre competição intrapopulacional, à medida que a população aumenta se observa a redução das taxas de crescimento. Nesse caso, ao invés de se observar um valor fixo de "r", esse decresce após determinado ponto. Este modelo de crescimento é denominado logístico (DIAS, 2018).

Nesse caso, a curva se apresenta de forma assíntota. No início, o crescimento é lento, esse acelera até o ponto de inflexão, que corresponde à metade da capacidade de suporte (normalmente designada como K) (NASCIMENTO et al.,2017). A partir do ponto de inflexão o crescimento desacelera até a população estabilizar no valor de K (CRUZ et al., 2016).

$\mathrm{Na}$ realidade, o comportamento da curva pode ser de diferentes formas. Esse irá depender dos atributos populacionais (valor de $\mathrm{r}$ ), bem como das características do ambiente (valor de K) (LEITÃO et al., 2018).

Como apresentado em seus estudos, Cruz et al. (2016) utilizaram os dois modelos, exponencial e o logístico, para análise de parâmetros populacionais da cidade de Gurupi. No modelo de Malthus, que considera como hipótese básica que a população cresce sem qualquer restrição, não admitindo 
fatores que regulam seu crescimento, o resultado da análise dos parâmetros está representado na Figura 1.

Figura 1 - Modelo exponencial para crescimento de uma população em condições ideais

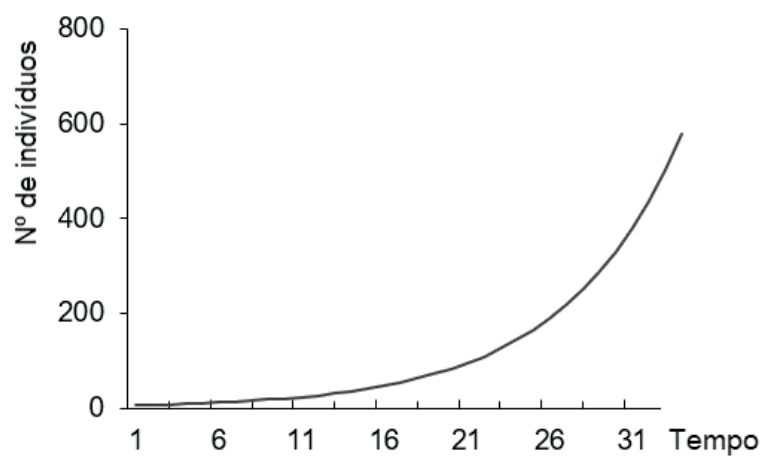

Fonte: Os autores.

No Modelo de Verhulst, em que a população sofre inibições no seu crescimento, ou seja, admite-se que a taxa de crescimento decresce com o aumento da população. $\mathrm{O}$ resultado da análise dos parâmetros está representado pela Figura 2.

Figura 2 - Modelo logístico para crescimento de uma população em condições ideais

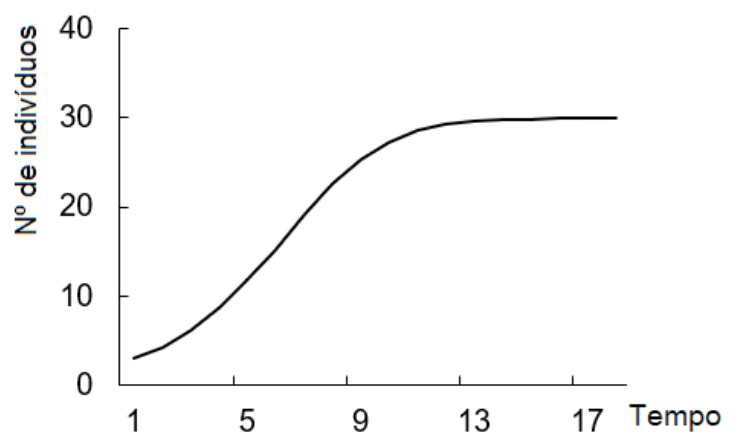

Fonte: Os autores.

\subsection{Interações populacionais}

As interações populacionais são as influências que os indivíduos ou grupos exercem uns sobre os outros. Dentro de uma população e entre populações da mesma ou de diferentes espécies. Podem ser divididas em intraespecíficas, quando ocorrem dentro de uma população e em interespecíficas, quando ocorrem em populações diferentes (LANDA, 2008). São divididas em favoráveis ou desfavoráveis, aquelas interações que favoreçam a abundância e as taxas elevadas de sobrevivência e o crescimento são consideradas favoráveis e aquelas que reduzam essas características são consideradas desfavoráveis (DEPRÁ et al., 2018).

Interações desfavoráveis tendem a ser minimizadas em favor da simbiose positiva, que melhora a sobrevivência das espécies interativas. São classificadas como interações desfavoráveis os casos de competição, de predação, de amensalismo e de parasitismo, quando pelo menos uma das espécies é prejudicada (SOLOMON, 1980). Populações com interações favoráveis têm um aumento em sua densidade populacional, como comensalismo, protocooperação e mutualismo (FARIAS et al. 2018).

Em ecossistemas marinhos, as principais populações de predadores são moldadas por fatores ambientais que afetam a abundância de presas. Acoplando estudos populacionais de predadores com registros independentes de abundância de presas se observa que as flutuações das presas afetam os parâmetros de fecundidade e a abundância de seus predadores (SILVA et al., 2014; FERREIRA et al., 2019).

\subsection{Dinâmica Populacional e as Mudanças Climáticas}

Problemas globais atuais, nomeadamente as alterações climáticas relacionadas com o aumento global da temperatura, têm causado preocupação e discussão em diversos níveis (KAHILAINEN et al.,2018). De acordo com Walter et al. (2018), populações de insetos exibem respostas variadas para a mudança global. As mudanças climáticas não são os únicos aspectos que interferem na dinâmica de população. A tendência de uma população aumentar ou diminuir, ao longo do tempo, indica mudanças que podem apresentar preocupação (ANDRADE et al., 2018).

Estudos de Kahilainen et al. (2018) comprovam que com as alterações antrópicas do ambiente, as mudanças climáticas interferem em metapopulações de borboletas. Essas metapopulações que habitam paisagens fragmentadas são mais vulneráveis às mudanças climáticas que outras populações. Dessa forma se pode então sugerir que a mudança climática pode aumentar o risco de extinção e, assim, alterar a sua dinâmica.

\section{Conclusão}

Com base nos artigos selecionados foi possível verificar a importância do uso da dinâmica populacional para alguns estudos. Uma população não é somente influenciada, essa também influencia seu habitat e outros indivíduos. O tema "dinâmica populacional" não é recente e os livros utilizados para essa pesquisa trazem, de forma bem descrita, todos os atributos necessários para se conhecer uma população, mas algumas vezes se percebem os erros básicos em conceitos e mesmo em alguns cálculos.

Observou-se que a maioria dos artigos, entre os que foram selecionados, retrata resultados de estudos sobre populações específicas, como tabelas de vida e formas de crescimento, que necessitam de informações para compreender o funcionamento da mesma, dessa forma, possibilitando gerenciar o tamanho das populações de espécies que possam vir a interferir em processos ecológicos, sociais ou econômicos. Ainda não existem dados reais sobre as populações naturais. São poucos os dados sobre as espécies e, para surpresa dos autores, praticamente inexistem dados reais de populações naturais do Brasil.

Por fim, compreende-se a relevância de se estudar uma população e se sugere, ainda, tomando como base dados revelados nos artigos pesquisados, a necessidade de 
novos estudos que ainda possuem lacunas, e esses estudos venham complementar e contribuir para o conhecimento de populações, incluindo aquelas que nem sempre se compõem por indivíduos e não organismos.

\section{Referências}

ANDRADE, B.S. et al. Efeitos das mudanças climáticas sobre as comunidades de peixes na Bacia Amazônica. Rev. Cien. Soc., v.2, n.4, p.107-124, 2018. doi: 10.30810/rcs.v2i4

AKCA, I. et al. Demographics and population projection of Aphis fabae (Hemiptera: Aphididae): with comments on the criteria for research in tables of life. J. Econ. Entomol., v.108, n.4, p.14661478, 2015. doi: 10.1093/jee/tov187.

ALVES, J.E.D. Demografia ecológica: população e desenvolvimento numa perspectiva ecocêntrica. Rev. Esp., v.7, n.1, p.36-45, 2018.

BARROS, C.L.; PEDRO, F.S.; ESMI, E. Modelos de dinâmica populacional para processos fuzzy autocorrelacionados. Proc. Ser. Braz. Soc. App. Comp. Math., v.6, n.1, 2018. doi: 10.5540/03.2018.006.01.0397

CAYUELA, H. et al. Multiple density dependent processes shape the dynamics of a spatially structured amphibian population. J. Anim. Ecol., v.88, p.164-177, 2018. doi: 10.1111/13652656.12906

COSTA, M.B. et al. Tabela de vida de fertilidade de Planococcus citri Costa, M. B. et al. (Risso) e Planococcus minor (Maskell) (Hemiptera: Pseudococcidae) em cafeeiro. Cof. Scien., v.11, n.2, p.204-210, 2016. doi: $10.25186 /$ cs.v11i2

CRUZ, P.A.; ROSA, L.P.; CRUZ, L.C.C. A utilização de modelos matemáticos para análise de parâmetros populacionais da cidade de Gurupi. Rev. Cereus., v.8, n.1, p.33-45, 2016. doi: 10.18605/2175-7275/cereus.v8n1p33-45

DALL, S. R. X. et al. An evolutionary ecology of individual differences. Ecol Lett., v.15, n.10, p.1189-1198, 2012. doi:10.1111/ j.1461-0248.2012.01846.x

DEPRÁ, M.S.; GAGLIANONE, M.C. Interações entre plantas e polinizadores sob uma perspectiva temporal. Oecol. Aust., v.22, n.1, p.1-16, 2018. doi: 10.4257/oeco.2018.2201.01

DIAS, F.H.S. Sincronismo em um novo modelo metapopulacional com migração independente da densidade. Proc. Ser. Braz. Soc. App. Comp. Math., v.6, n.1, p.1-7, 2018. doi: 10.5540/03.2018.006.01.0432

FARIAS, A.P. et al. Dinâmica populacional e parasitismo natural de Diaphorina citri Kuwayama (Hemiptera: Liviidae) em pomares de citros em Sergipe. Entom. Bras., v.11, n.1, p.20-25, 2018. doi: 10.12741/ebrasilis.v11i1.720

FERREIRA, M.S.N., DUARTE, M.R., SILVA, E.P. Recursos pesqueiros de mariscagem: uma revisão da literatura sobre Iphigenia brasiliensis. Ens. Cienc., v.23, n.1, p.24-34, 2019. doi: 10.17921/1415-6938.2019v23n1p24-34.

FONTANA, R.L.M. et al. Teorias demográficas e o crescimento populacional no mundo. Ciênc. Human. Soc. Unit., v.2, n.3, p.113-124, 2015 .

GAVINA, M.K.A. et al. Multi-species coexistence in Lotka Volterra competitive systems with crowding efects. Scien. Rep., v.8, n.1198, p.1-8, 2018. doi: 10.1038/s41598-017-19044-9.

GIRÃO FILHO, J.E. et al. Exigências térmicas e tabelas de esperança de vida e fertilidade de Aphis craccivora (Hemiptera: Aphididae) em Vigna unguiculata (Fabales: Fabaceae) sob condições de laboratório. Arq. Inst. Biol., v.86, 1-9, 2018, doi:

\subsection{0/1808-1657000142018.}

GOMES, L.C. Síndromes de dispersão do estrato arbóreoarbustivo em dois fragmentos florestais do Pantanal Sul, MS. Rev. Biodivers., v.17, n.2, p. 139-149, 2018.

HASSAN, S.S. et al. Computational dynamics of the NicholsonBailey models. Euro. Phys. J. Plus., v.133, n.349, p.1-22, 2018. doi: 10.1140/epjp/i2018-12164-1

KAHILAINEN, A. et al. Metapopulation dynamics in a changing climate: Increasing spatial synchrony in weather conditions drives metapopulation synchrony of a butterfly inhabiting a fragmented landscape. Glob. Chan. Biol., v.24, n.9, p.4316-4329, 2018. doi: $10.1111 / \mathrm{gcb} .14280$

LANDA, G.G. Ecologia: uma ciência complexa vista sob uma linguagem simples uma forma de entender e conservar o meio ambiente. Belo Horizonte: FUMARC, 2008.

LEITÃO, M.L.C. et al. Diferenças ecomorfológicas e funcional em populações de phalloceros harpagos Lucinda, 2008, na bacia do Alto Rio Paraná. Biot. Amazon., v.8, p.34-38, 2018. doi: 10.18561/2179-5746/biotaamazonia.v8n3p34-38

LEVINS, R. Some demographic and genetic consequences of environmental heterogeneity for biological control. Bull. Entomol. Soc. Am., v.15, p.237-240, 1969.

MAIA, B.M.C. et al. Registro de forésia de ovos de Dermatobia hominis (L.) (Diptera, Oestridae) por Fannia canicularis (L.) e Fannia punctipennis (Albuquerque) (Diptera, Fanniidae) em São Paulo, Brasil. Entom. Bras., v.11, n.3, p.226-229, 2018. doi: 10.12741/ebrasilis.v11i3.780

MARQUES, C.C. et al. Biologia e tabela de vida do ácaro predador Euseius concordis (CHANT, 1959) (acari: phytoseiidae) em pinhão-manso. Rev. Caating., v.28, n.2, p.249-255, 2015.

MALTHUS, T.R. An essay on the principle of population. London: J. Johnson, 1798.

MEDEIROS, M.O. et al. Tabela de vida de fertilidade para Scaptocoris carvalhoi BECKER, 1967 (Hemiptera: Cydnidae) em Urochloa decumbens (POACEAE). Biodivers., v.16, n.3, p.1$15,2017$.

NASCIMENTO, I.S. et al. Variação sazonal e interanual na dinâmica populacional de Croton blanchetianus em uma floresta tropical seca. Pesq. Flor. Bras., v.37, n.92, p.437-446, 2017. doi: 10.4336/2017.pfb.37.92.1191

OLIVEIRA, R.L.C.; STOFFEL, N.M.; FARIAS, H.L.S. Estrutura populacional e distribuição espacial de alexa canaracunensis em uma floresta estacional semidecidual submontana no norte de Roraima, Amazônia brasileira. Rev. Eletr. Casa Maku., v.1, n.2, p.100-109, 2018. doi: 10.24979/iss2pp79

OVASKAINEN, O.; I. HANSKI. Spatially structured metapopulation models: global and local assessment of metapopulation capacity. Theor. Popul. Biol., v.60, p.281-302, 2001.

PELLI, A. et al. Parâmetros populacionais para Triatoma sordida Stal, 1859, o vetor mais frequente da doença de Chagas no Triângulo Mineiro (Heteroptera, Triatominae). Rev. Soc. Bras. Med. Trop., v.40, n.1, p.1-4, 2007. doi: 10.1590/S003786822007000100005

PRADEU, T. Organisms or biological individuals? Combining physiological and evolutionary individuality. Biol. Philos., v.31, n.6, p.797-81, 2016. doi: 10.1007/s10539-016-9551-1f.

RICKFLES, R.E. A economia da natureza. Rio de Janeiro: Guanabara Koogan, 2010.

RODRIGUES-SOARES, F. et al. Genetic structure of 
pharmacogenetic biomarkers in Brazil inferred from a systematic review and population-based cohorts: a RIBEF/EPIGEN-Brazil initiative. Pharm. J. v.18, n.6, p.749-759, 2018. doi: 10.1038/ s41397-018-0015-7.

ROUGHGARDEN, J. Production function from ecological populations: a survey with emphasis on spatially implicit models. In: TILMAN, D.; KAREIVA, P. Spatial ecology. Princeton: Princeton University Press, 1997. p.296-317.

SANTOS, R.G.; FRANCHIN, A.G.; NUNES, J.F. Frugivoria por aves em Cecropia pachystachya Trécul (urticaceae) em uma área verde urbana de Passos - MG. Biodivers., v.16, n3, p.16-26.2017.

SILVA, C.V. et al. Pesca artesanal e cetáceos que ocorrem no litoral leste do Rio de Janeiro: uma abordagem etnoecológica para verificar a existência de manejo tradicional. Bol. Inst. Pesca, v.40, n.4, p.521-539, 2014.

SILVA, J.M.C. Modelagem ambiental e a conservação da biodiversidade. Megadiversidade, v.3, n.1/2, p.101, 2007.

SILVA, L.C. et al. Parâmetros demográficos do Aphis craccivora Koch, 1854 em cultivares e variedades crioulas de Vigna unguiculata (L.) Walp) da região amazônica. Act. Igua., v.7, n.5, p.109-119, 2018.

SILVA, L.S.B. et al. Modelo entomológico determinístico sob efeito da pluviosidade para o Aedes aegypti e o Aedes albopictus. Tend. Mat. Aplic. Comp., v.19, n.2. p.289-303. 2018. doi: 10.5540/ tema.2018.019.02.0289

SILVA, M.O.; ROSA, J. Modelos de sistemas dinâmicos aplicados à ecologia: Malthus, Verhulst e Lotka-Volterra. Rev. Inic. Cient.IFPR, v.4, n.1., p.64-78, 2018.

SOBY, S.T.M., ESTER, B. Agricultural development models in the age of limits. J. Agric. Environ. Ethics., v.30, n.1, p.87-98, 2017. doi: $10.1007 / \mathrm{s} 10806-017-9655-\mathrm{x}$

SOlOMON, M.E. Dinâmica de populações. São Paulo: Pedagógica e Universitária, 1980.

SOUZA, S.C.P.M. et al. A. Dinâmica populacional de doze espécies arbóreas de diferentes grupos ecológicos em um trecho de Floresta Ombrófila Densa Submontana. Scien. Plen., v.14, n.5, p.1-15, 2018. doi: 10.14808/sci.plena.2018.052401.

SUTER II, G.W.; NORTON, S.B.; FAIRBROTHER, A. Individuals versus organisms versus populations in the definition of ecological assessment endpoints. Integr. Environ. Assess. Manag., v.1, n. 4, p. 397-400, 2005.

WALTER, J.A. et al. Life history and habitat explain variation among insect pest populations subject to global change. Ecosph., v.9, n.5, p.1-13, 2018. doi: 10.1002/ecs2.2274 